\title{
BMJ Open Assessment of cardiac resynchronisation therapy in patients with wide QRS and non-specific intraventricular conduction delay: rationale and design of the multicentre randomised NICD-CRT study
}

\author{
Romain Eschalier, ${ }^{1}$ Sylvain Ploux, ${ }^{2}$ Bruno Pereira, ${ }^{3}$ Nicolas Clémenty, ${ }^{4}$ \\ Antoine Da Costa, ${ }^{5}$ Pascal Defaye ${ }^{6}$ Stéphane Garrigue, ${ }^{7}$ Jean-Baptiste Gourraud, ${ }^{8}$ \\ Daniel Gras, ${ }^{9}$ Benoît Guy-Moyat, ${ }^{10}$ Christophe Leclercq, ${ }^{11}$ Pierre Mondoly, ${ }^{12}$ \\ Pierre Bordachar ${ }^{2}$
}

To cite: Eschalier R, Ploux S, Pereira B, et al. Assessment of cardiac resynchronisation therapy in patients with wide QRS and non-specific intraventricular conduction delay: rationale and design of the multicentre randomised NICD-CRT study. BMJ Open 2016;6: 012383.

doi:10.1136/bmjopen-2016012383

- Prepublication history for this paper is available online. To view these files please visit the journal online (http://dx.doi.org/10.1136/ bmjopen-2016-012383).

Received 22 April 2016 Revised 13 September 2016 Accepted 14 September 2016

CrossMark

For numbered affiliations see end of article.

Correspondence to Pr Romain Eschalier; reschalier@chuclermontferrand.fr

\section{ABSTRACT}

Introduction: Cardiac resynchronisation therapy (CRT) was initially developed to treat patients with left bundle branch block (LBBB). However, many patients with heart failure have a widened QRS but neither left-BBB nor right-BBB; this is called non-specific intraventricular conduction delay (NICD).

It is unclear whether CRT is effective in this subgroup of patients.

Methods and analysis: The NICD-CRT study is a prospective, double-blind, randomised (1:1), parallelarm, multicentre trial comparing the effects of CRT in patients with heart failure, a reduced left ventricular ejection fraction (LVEF $<35 \%$ ) and NICD, who have been implanted with a device (CRT-pacemaker or CRT-defibrillator) that has or has not been activated. Enrolment began on 15 July 2015 and should finish within 3 years; 40 patients have already been randomised and 11 centres have agreed to participate. The primary end point is the comparison of the proportion of patients improved, unchanged or worsened over the subsequent 12 months. 100 patients per group are required to demonstrate a difference between groups with a statistical power of $90 \%$, a type I error of $0.05 \%$ (two-sided) and a loss to follow-up of $10 \%$. This trial will add substantially to the modest amount of existing data on CRT in patients with NICD and should reduce uncertainty for guidelines and clinical practice when added to the pool of current information.

Ethics and dissemination: Local ethics committee authorisations have been obtained since May 2015. We will publish findings from this study in a peer-reviewed scientific journal and present results at national and international conferences.

Trial registration number: NCT02454439; pre-results.

\section{Strengths and limitations of this study}

- This is the first prospective study designed to evaluate the effectiveness of cardiac resynchronisation therapy (CRT) in patients with nonspecific intraventricular conduction delay (NICD).

- The strong design of NICD-CRT study may help to give a clear answer to this crucial question.

- The NICD-CRT study is a multicentre study involving teams with high experience in CRT trials and cardiac rhythm management.

- An experimented ECG CoreLab may avoid bias of ECG pattern recruitment.

- Including patients with NICD may be a limitation since it is difficult sometimes to differentiate between left bundle branch block and NICD patterns.

\section{INTRODUCTION}

Several randomised control trials have found $^{1-4}$ cardiac resynchronisation therapy (CRT) to be beneficial in heart failure (HF) patients with reduced left ventricular ejection fraction (LVEF) and prolonged QRS duration. The concept of resynchronisation therapy is challenged by the observation that for patients with similar QRS duration, those with left bundle branch block (LBBB) respond significantly better than patients with non-specific intraventricular conduction delay (NICD). ${ }^{5}{ }^{6}$ An important issue is to identify such patients with NICD pattern since current guidelines use a definition by default and that this population represents a 
large proportion of HF patients with reduced ejection fraction and wide QRS $(6.1-30.3 \%$ in dilated cardiomyopathy) ${ }^{7-9}$ The American Heart Association/American College of Cardiology Foundation/Heart Rhythm Society (AHA/ACCF/HRS) recommendations, ${ }^{10}$ published in 2009, specified that non-specific or unspecified intraventricular conduction disturbance is defined by "a QRS duration greater than $110 \mathrm{~ms}$ in adults (...) without meeting the criteria for RBBB or LBBB. The definition may also be applied to a pattern with RBBB criteria in the precordial leads and LBBB criteria in the limb leads, and vice versa". Furthermore, patients with NICD have a poorer prognosis since the presence of NICD in patients with or without heart disease is associated with an increased risk of death. ${ }^{1-15}$ NICD delay is observed in a variety of cardiac diseases (eg, ischaemic, hypertensive) and results obtained following CRT have only been assessed on limited sample sizes, without dedicated randomised studies. ${ }^{16}$ Moreover, the observed results have at times been conflicting. ${ }^{6} 1718$ The latest international guidelines tend to restrict the indications in this setting and the question arises as to whether to continue to implant HF patients with NICD. However, these guidelines are based on retrospective subgroup analyses ${ }^{19}$ and do not distinguish between RBBB and NICD. A dedicated prospective study is therefore warranted in order to assess the clinical effectiveness of CRT in HF patients with NICD pattern.

\section{METHODS AND ANALYSIS}

As highlighted above, there is currently uncertainty as to whether a CRT device implantation is beneficial in patients with NICD given the absence of dedicated prospective, randomised, blinded trials in this setting. The present NICD-CRT clinical trial was designed as a study to investigate whether a CRT device implantation may be beneficial in patients with wide QRS and NICD pattern.

The study is a prospective, controlled, two-parallel arm, randomised, therapeutic, double-blind design and multicentric clinical trial comparing a CRT-D (defibrillator) or CRT-P ( pacemaker) ON group versus CRT-D or
CRT-P OFF group in HF patients with NICD and reduced ejection fraction (figure 1).

\section{Objectives}

\section{Primary objective}

To assess the clinical effectiveness of CRT in HF patients with NICD and reduced ejection fraction on a 12-month HF status.

\section{Secondary objectives}

To assess the impact of CRT in HF patients with NICD and reduced ejection fraction on all-cause, cardiac and HF deaths and hospitalisations, quality of life, functional capacity and reduction in left ventricular (LV) volumes at 6 and 12 months.

\section{Study end points}

\section{Primary end point}

The comparison in terms of z-score defined as a composite of two clinical end points (12 months all-cause deaths and percentage of hospitalisations for $\mathrm{HF}$ at 12 months) combined using an average z-score. This framework allows the combination of multiple clinical end points (especially time-to-event end points) into a single statistical assessment, without assigning a rank of relative importance to each domain as is required with other related methods (ref). Time-to-event variables will be first transformed into log-rank scores and then converted to z-scores by subtracting the mean and dividing by the SD of the pooled data. The z-scores are then aligned to the same direction so that worse outcomes have smaller scores. The z-scores will be then averaged across end points for each patient. Treatment groups will be compared with respect to this average $z$-score.

\section{Secondary end points}

- Twelve-month deaths (HF, cardiovascular and allcause deaths),

- Quality-of-life questionnaires at 6 and 12 months:

- Minnesota Living With Heart Failure Questionnaire (MLWHFQ): improvement of at least 20 points

- Functional capacity at 6 and 12 months:

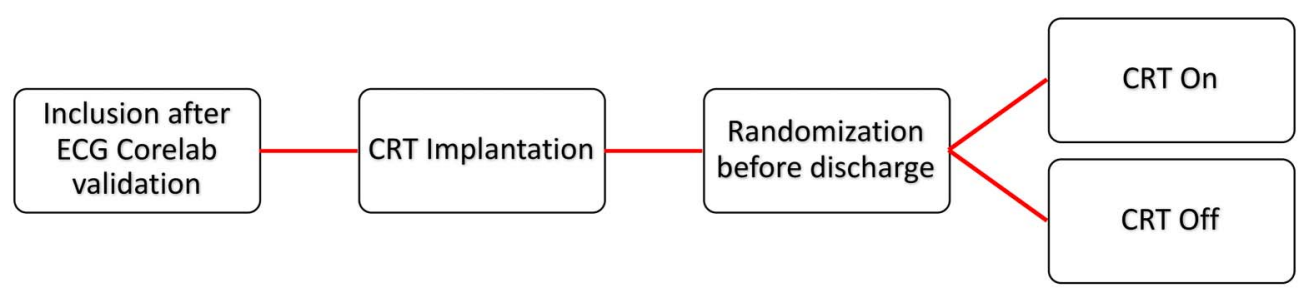

Figure 1 Flow chart describing NICD-CRT. After verification of eligibility and signing of the informed consent form, CRT device implantation will be performed according to current clinical practice. Initially, quadripolar LV lead will be used in the present study. In case of failure of quadripolar LV lead implantation, a bipolar LV lead may be used. Randomisation will be performed following the inclusion consultation. Follow-up will be the same in both groups: inclusion/baseline visit, and M6-12 visits in the investigation centre. LV, left ventricular; NICD-CRT, non-specific intraventricular conduction delay/cardiac resynchronisation therapy. 
- New York Heart Association (NYHA) classification reduction $\geq 1$ class, ${ }^{20}$

- Six-minute walk test improvement of at least $10 \%$ in distance, ${ }^{20}$

- Peak oxygen consumption increased by $1.0 \mathrm{~mL} /$ $\mathrm{kg} / \mathrm{min}^{21}$

- Percentage of hospitalisations for HF, for cardiovascular reasons and for all causes at 6 and 12 months,

- Decrease $>15 \%$ in end-diastolic and/or end-systolic volumes of the left ventricle at 6 and 12 months. ${ }^{22} 23$

\section{Trial inclusion/exclusion criteria}

The patients must fulfil the following criteria before their enrolment in the trial:

- Patients over 18 years old

- NYHA class II to IV ambulatory

- QRS duration $>130 \mathrm{~ms}$

- Patients in sinus rhythm

- $\mathrm{LVEF}<35 \%$

- QRS morphology: NICD according to the AHA/ ACCF/HRS recommendations ${ }^{10}$ (non-LBBB and non-RBBB):

- Absence of broad notched or slurred $\mathrm{R}$ wave in leads I, aVL, V5 and V6;

- Presence of a $Q$ wave in leads I, V5, V6;

- No rsr', rsR' or rSR' pattern in leads V1 or V2.

- Life expectancy expected to exceed 1 year with good functional status

- Optimal pharmacological therapy of HF according to the attending clinician.

Patients will be excluded from the trial for the following reasons:

- Inability to understand or decline the study,

- Impaired mobility,

- Inability to fill out the questionnaire independently,

- Patients with permanent atrial fibrillation,

- Pregnant women,

- Dependent adults,

- Life expectancy $<1$ year due to causes other than HF.

\section{Study protocol}

Patients meeting the inclusion criteria, admitted to each cardiology department (University Hospital, ClermontFerrand, France; University Hospital, Bordeaux, France; University Hospital, Saint-Etienne, France; University Hospital, Grenoble, France; University Hospital, Limoges, France; University Hospital, Nantes, France; Saint-Augustin Clinic, Bordeaux, France; Nouvelles Cliniques Nantaises, Nantes, France; University Hospital, Tours, France; University Hospital, Toulouse, France; University Hospital, Rennes, France) for CRT device implantation with NICD pattern (de novo or upgrading without previous chronic right ventricular pacing indications) will be screened. Eligible patients will receive a clear verbal explanation and a written information sheet by the physicians. Finally, written consent of each patient (records stored by the promoter in a dedicated archives room) will be obtained.
An ECG CoreLab will review each ECG and validate the NICD pattern, before inclusion and CRT device implantation, according to AHA/ACCF/HRS recommendations ${ }^{10}$ ( figure 2).

After verification of patient's eligibility and the signing of the informed consent form, randomisation will be performed following the inclusion consultation. An automatic randomisation (stratified for QRS duration: $130-150 \mathrm{~ms}$ vs $>150 \mathrm{~ms}$, gender and investigator centre) will ensure balance between the two groups by minimisation with hierarchical stratification, ${ }^{24}$ using Stata software by a statistician independent of the protocol. An immediate answer, sent by secured email, will specify the patient's randomisation group (CRT ON vs CRT OFF) and inclusion code.

A CRT system will be implanted in all patients and will be activated (CRT ON) or inactivated (CRT OFF) after randomisation (data will be transmitted by an assistant of the clinical researcher who is not in direct contact with the patient). CRT device implantation will be performed according to the standard practice of each clinician. The decision to implant a pacemaker or a defibrillator associated with the CRT system will be made by the interventional cardiologist according to guidelines, age of the patient, aetiology of the heart disease and habits. Initially, a quadripolar LV lead will be used in the present study. In case of failure of quadripolar LV lead implantation, a bipolar LV lead may be used. The position of the LV lead will depend on coronary venous anatomy, lead stability and pacing threshold (sites with phrenic nerve capture will be avoided). Remote monitoring will be activated systematically in all included patients prior to hospital discharge.

Antitachycardia treatments (in case of implanted defibrillator) will be programmed at the discretion of the clinicians.

However, the two groups will be programmed as follows with regard to pacing modes:

- In the CRT ON group: DDD biventricular mode; clinicians will define the best atrioventricular (80$150 \mathrm{~ms})$ and interventricular (0-40 ms) delays and the minimal heart rate for each patient;

- In the CRT OFF group: back-up stimulation mode: VVI $40 \mathrm{bpm}$ (right ventricular pacing only).

No optimisation of $\mathrm{A}-\mathrm{V}$ and $\mathrm{V}-\mathrm{V}$ delay are planned in this NICD-CRT study since the European guidelines do not recommend such optimisation. ${ }^{25}$

\section{Patient follow-up}

Follow-up will be the same in groups: inclusion/baseline visit, and M6-12 visits in the investigation centre. The planned data collection is summarised in table 1 . At baseline, clinical examination (NYHA class, quality of life, 6 min walk test, blood pressure, aetiology of heart disease, peak and threshold oxygen consumption), ECG, biological sample (haemoglobin, kalemia, serum sodium, modification of diet in renal disease and brain natriuretic peptide), transthoracic echocardiography 


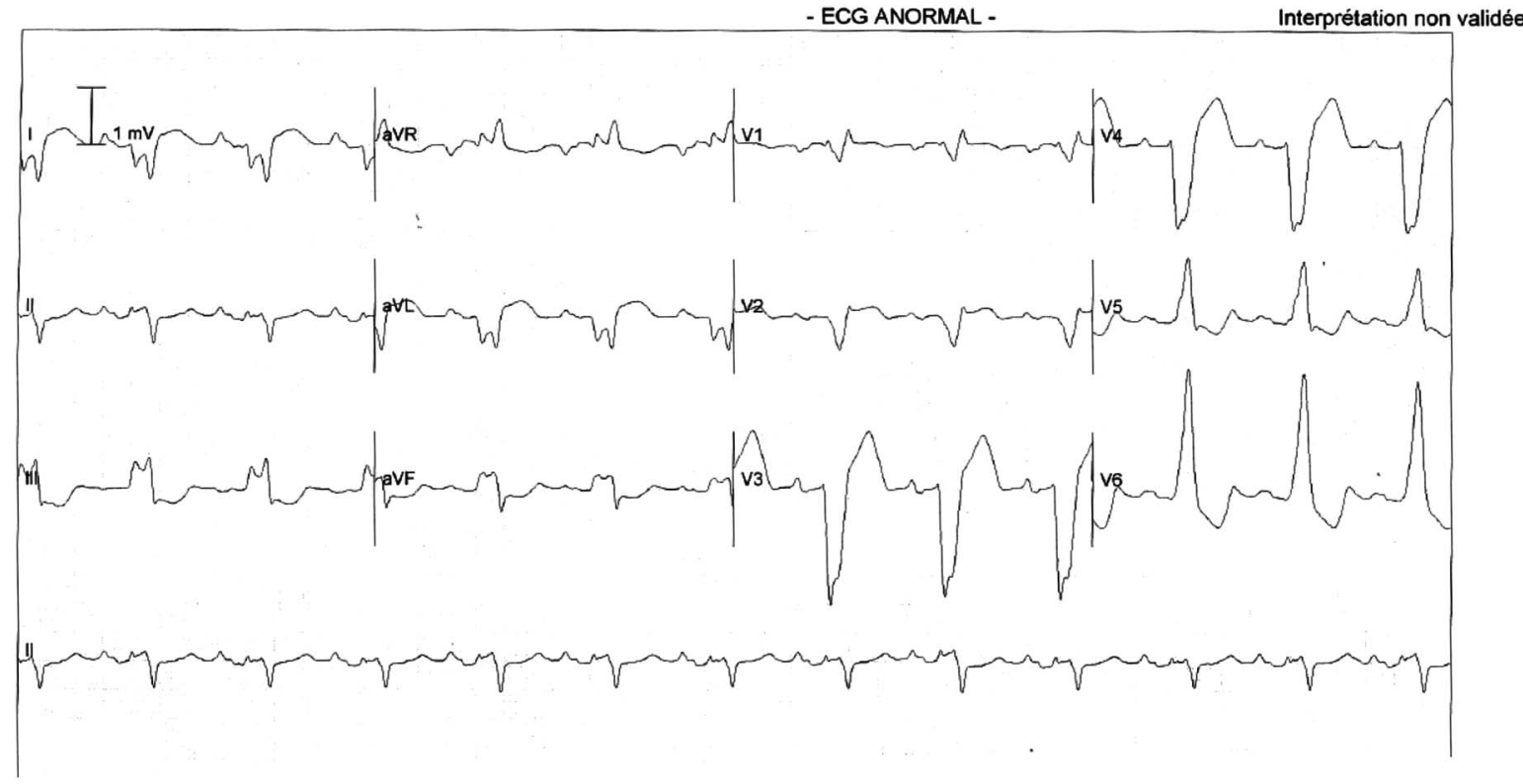

Figure 2 Example of ECG of a NICD pattern. ECG of a 65-year-old woman with a induced chemotherapy cardiomyopathy and a LV ejection fraction of $27 \%$. On the ECG, the rS pattern in lead I and aVL are criteria against the diagnosis of LBBB. LBBB, left bundle branch block; LV, left ventricular; NICD, non-specific intraventricular conduction delay.

(LVEF, LV dimensions and volumes, Strain) and myocardial MRI will be performed. The same evaluation (clinical examination and different examinations) will be performed at 6 and 12 months associated with device interrogation. All these tests will be performed by a clinical research assistant and the physicians will be blinded to the randomisation group.

\section{Sample size calculation}

Owing to lack of data and reported discrepancies in the literature regarding the impact of CRT in patients with NICD, ${ }^{6} 1718$ proposing an accurate sample size estimation appeared a priori difficult. In a previous review, we had shown that the number of patients with NICD pattern included in the major CRT trials showed considerable variation, ranging from $1 \%$ in the MIRACLE trial to $21 \%$ in the REVERSE study, that is, from $961 / 5245$ patients. ${ }^{16}$ The number of participants to be included in the present pilot study has been extrapolated from data obtained in previous works, according to which 100 patients per group will be included. For a two-sided type I error at 5\% and a statistical power equals $90 \%, n=86$ randomised patients will allow highlighting a clinical and realistic effect-size of 0.5 which could be considered significant according to Cohen's recommendations (Statistical power analysis for the behavioural sciences (2nd edn). New Jersey: Lawrence Erlbaum, 1988) which has defined effect-size bounds as: small (ES: 0.2), medium (ES: 0.5 ) and large (ES: 0.8, 'grossly perceptible and therefore large'). To take into account loss to follow-up (around 10\%) and to maximise the statistical power, the inclusion of 100 patients per group was proposed. For 100 evaluable patients (50 per group), a difference in Z-score between the two arms will be considered

\begin{tabular}{|c|c|c|c|}
\hline Testing or evaluation & Baseline & $\begin{array}{l}\text { Six } \\
\text { months }\end{array}$ & $\begin{array}{l}\text { Twelve } \\
\text { months }\end{array}$ \\
\hline Physical examination & $\checkmark$ & $\checkmark$ & $\checkmark$ \\
\hline NYHA Class & $\checkmark$ & $\checkmark$ & $\checkmark$ \\
\hline 6 min walking test & $\checkmark$ & $\checkmark$ & $\checkmark$ \\
\hline 12-lead ECG & $\checkmark$ & $\checkmark$ & $\checkmark$ \\
\hline $\begin{array}{l}\mathrm{Hb}, \mathrm{Na}, \mathrm{K} \\
\text { creatininaemia, } \\
\text { NTproBNP }\end{array}$ & $\checkmark$ & $\checkmark$ & $\checkmark$ \\
\hline QOL & $\checkmark$ & $\checkmark$ & $\checkmark$ \\
\hline $\begin{array}{l}\text { Transthoracic } \\
\text { echocardiography }\end{array}$ & $\checkmark$ & $\checkmark$ & $\checkmark$ \\
\hline $\begin{array}{l}\text { Peak and threshold } \\
\text { oxygen consumption }\end{array}$ & $\checkmark$ & $\checkmark$ & $\checkmark$ \\
\hline Medication & $\checkmark$ & $\checkmark$ & $\checkmark$ \\
\hline Cardiac MRI & $\checkmark$ & & \\
\hline $\begin{array}{l}\text { All-cause, HF and } \\
\text { cardiac deaths }\end{array}$ & & $\checkmark$ & $\checkmark$ \\
\hline $\begin{array}{l}\text { All-cause, HF and } \\
\text { cardiac hospitalisations }\end{array}$ & & $\checkmark$ & $\checkmark$ \\
\hline $\begin{array}{l}\text { CRT device } \\
\text { interrogation }\end{array}$ & & $\checkmark$ & $\checkmark$ \\
\hline \multicolumn{4}{|c|}{$\begin{array}{l}\text { CRT, Cardiac resynchronisation therapy; Hb, haemoglobin; HF, } \\
\text { heart failure; K, kalaemia; Na, serum sodium; NT-ProBNP, } \\
\text { N-terminal portion of pro brain natriuretic peptide; NYHA, } \\
\text { New York Heart Association; QoL, quality of life. }\end{array}$} \\
\hline
\end{tabular}

significant for an adjusted type I error as determined by the Land and DeMets method (East software).

\section{Statistical analyses}

All analyses will be conducted with Stata statistical software, V.13 (StataCorp LP, College Station, Texas, USA). 
A two-sided $p$ value of $<0.05$ will be considered to indicate statistical significance. Statistical analysis will be conducted on an intention-to-treat (ITT) basis. The continuous variables will be presented as their means and SDs or as medians and IQRs according to statistical distribution (Shapiro-Wilk test). The categorical parameters will be expressed as the number of patients and associated percentages. The patients will be described and compared at inclusion according to the following variables: compliance with the eligibility criteria, epidemiological characteristics, clinical and biological characteristics and treatment characteristics. Student's t-test or Mann-Whitney $\mathrm{U}$ test if t-test's assumptions not met ((1) normality studied using Shapiro-Wilk test and (2) homoscedasticity verified by Fisher-Snedecor test) was applied to compare primary end point (Z-score) between randomised groups. Adjusted analysis will be performed with the use of random-effects linear regression model in order to (1) take into account adjustment on possible confounding covariates selected according to clinical relevance (notably parameters considered for stratification) and (2) consider within-centre and between-centre variability. All effect sizes will be presented with $95 \%$ CIs. Other continuous variables (eg, 6 min walk test, number of readmissions for all causes and for HF, biomarkers for $\mathrm{HF}$ and QoL at 12 months on MLWHFQ and Short Form 12) will be compared similarly with the use of the unpaired t-test or the Mann-Whitney U test when appropriate. Adjusted analyses will be performed using the same adjustment variables described above. The $\chi^{2}$ test (or Fisher's exact test as appropriate) will be used for secondary categorical outcomes (eg, NYHA classification). The timeto-event curves will be calculated with the use of the Kaplan-Meier method. Comparisons of censored data will be conducted in univariate analysis by log-rank test and in multivariate analysis by the Cox proportional regression model. The statistical analyses concerning components of composite z-score were performed with a corrected type I-error taking into account multiple comparisons. Longitudinal analysis using mixed models will be used to take into account between-patient and within- patient variability. If the frequency of missing data is $>5 \%$, an additional analysis will be performed using the multiple imputation method (Stata command $\mathrm{mi})$.

\section{Data monitoring safety committee}

A Data Monitoring Safety Committee (independent from the sponsor) will meet initially at the launch of the study and thereafter throughout the study, on its own initiative or at the request of the sponsor, and will discuss the results of the intermediate analyses. It will have a consultative role. It will provide a general opinion on the progress of the study as well as provide input in helping make difficult decisions during the course of the study for which an independent judgement is desirable. The Data Monitoring Safety Committee will decide on possible early termination in light of data transmitted by the adjudication committee, the role of which will be defined by the Data Monitoring Safety Committee. The study will be terminated if the difference between the two groups becomes excessive.

\section{Data storage and management}

Data storage and management will be performed according to international guidelines relevant in French institutions, as previously described. ${ }^{26}{ }^{27}$ All data will be entered using electronic case report form (created by Medsharing society) and data accuracy will be analysed by the NICD-CRT data manager. Data quality control measures will include queries to identify outliers and missing data. Only the research assistant and NICD-CRT study principal investigator (PI) will have access to protected personal health information. After inclusion, a unique identifier (linked to the participant's medical record number and a hard copy roster) will be stored in a locked cabinet in the PI's locked private office). The PI will ensure that the anonymity is preserved. The study PI will have access to the final trial data set, as will a biostatistician.

An adverse event (AE) is defined as any untoward medical occurrence which has occurred in a patient taking part in the study. A serious $\mathrm{AE}$ is defined as any untoward occurrence that: requires hospitalisation or prolongation of existing hospitalisation; results in persistent or significant disability; is life threatening; results in death or is otherwise considered medically significant by the investigator. $\mathrm{AE}$ will be recorded on an $\mathrm{AE}$ form. An assessment of the gravity, possible causality and expectedness event/reaction will be undertaken. At each visit, participants will be asked whether they have had any problems and if any AEs have occurred. The $\mathrm{AE}$ reaction reporting period begins for NICD-CRT trial when the participant is randomised and ends 12 months after the randomisation. All recorded $\mathrm{AE}$ will be followed up until they are resolved or the patient's participation in NICD-CRT trial ends. Resolution of these events will be entered on the AE form. Potential complications associated with the CRT device implantation (only potential serious $\mathrm{AE}$ in the present study, but however not dependent of the present study) are: pain, haematoma, infection, pericardial effusion and LV lead dislodgement.

The promoters of the NICD-CRT study have initially decided that the level of monitoring is low, which represent two visits/centre during the study after the beginning of the inclusion ( 1 nearly after the beginning of the active recruiting phase and 1 at the end of the inclusion).

\section{Ethics and dissemination}

The NICD-CRT study is currently in an active recruiting phase since the 15 July 2015 (first inclusion). The present study complies with the Declaration of Helsinki. Study completion date is estimated at July 2018. This 
multicentre, randomised, parallel-group trial has been registered at http://www.clinicaltrials.gov under the registration number NCT02454439. All the 11 centres have been activated since July 2015 (the last one in May 2016). Forty-one patients have been randomised and included in the NICD-CRT study. We will publish findings from this clinical study in a peer-reviewed scientific journal and present results at national and international conferences. All the centres have a good reputation in CRT clinical trials. A total of 200 patients have to be recruited in 24 months $(0.7$ patient/month/centre).

The statistical analysis plan and subsequent versions will be maintained in the study file. The statistical analysis plan may be revised during the study to take into account any changes to the protocol or other changes to the study that may have an impact on the statistical analysis initially planned. Any changes to the statistical plan or protocol analysis itself will be subject to the opinion of the local ethics committee and the promoter and communicated to the investigators (emails, newsletter and/or postal letter).

\section{DISCUSSION}

The NICD-CRT trial will examine whether the implantation of a CRT device in patients with NICD pattern and heart failure with reduced LV ejection fraction will improve the clinical status of this population.

The majority of patients previously included in the main CRT clinical trials also included patients with LBBB, since this therapy had been initially proposed to specifically target the detrimental impact of the LBBB-ventricular activation sequence. However, patients with NICD pattern represent a large proportion of patients with $\mathrm{HF}$ and reduced ejection fraction. Important discrepancies have been described in several studies regarding the potential benefit of CRT in patients with NICD pattern. Current results are moreover conflicting with, on the one hand, a reported benefit in terms of quality of life, exercise time and peak oxygen consumption in the MIRACLE ${ }^{28}$ trial, while, on the other, a reported lack of benefit in terms of clinical composite score, LV remodelling and mortality observed in other studies. ${ }^{6} 1718$ A recent meta-analysis suggested that CRT had a neutral effect in patients with NICD $^{5}$ with observed outcomes being poorer than in patients with LBBB. Furthermore, the findings regarding the clinical benefit of CRT-D in LBBB, but not in non-LBBB patients, were consistent throughout the Multicenter Automatic Defibrillator Implantation Trial (MADIT)CRT population evaluated by age, sex, NYHA class, ischaemic or non-ischaemic origin, QRS duration and baseline haemodynamic status, ${ }^{3}$ and recently confirmed by the long-term ( 7 years) analysis of the MADIT-CRT ${ }^{29}$ study. Several mechanisms may explain the apparent neutral effect of CRT in patients with non-LBBB: (1) the high prevalence of ischaemic cardiomyopathy in the NICD group, a factor known to adversely affect prognosis; ${ }^{28}{ }^{30}$ (2) a lesser amount of electrical dyssynchrony compared to patients with LBBB $^{31}$ and (3) the commonly performed aggregation of RBBB and patients with NICD in these subgroup analyses which precludes any firm conclusions regarding the effectiveness of CRT in these groups. Our observations obtained from epicardial electrical activation sequences ${ }^{31}{ }^{32}$ are in strong opposition with the recent results of the IPD meta-analysis, which consider QRS duration as the only indicator of CRT response and that QRS morphology does not provide additional information. ${ }^{33}$

In addition, current data analysing the impact of CRT in patients with NICD only stem from subanalysis studies. The relevance of subgroup analyses should be questioned however and the limited number of patients included in these studies is insufficient to draw any firm conclusions. Furthermore, no distinction was made between RBBB and NICD in the guidelines, which is somewhat surprising given the fact that electrical activation sequences and CRT effectiveness may differ between these two patient groups. While there are a number of limitations, the above results have nonetheless led to significant changes in the latest international guidelines, such that the pattern of the QRS complex and not solely QRS width has become a selection criterion for candidates for CRT device implantation. ${ }^{25} 34$

The discrepancies in the results obtained from different subanalyses thus warrant performing a dedicated randomised, controlled, double-blinded study to assess whether CRT may be also beneficial for HF patients with NICD and systolic dysfunction.

In the present trial, it was decided to use a Z-score for primary end point since Sun $e t a l^{35}$ have demonstrated that the Z-score enables detection of therapeutic efficacy (ie, CRT in the present study) using sample sizes of 100-150 patients per group, approximately double the power achievable assessing the effects. A physician blinded to CRT mode (CRT ON group or CRT OFF group) will perform patient's clinical assessment so as to limit any potential evaluation bias. In order to provide a comprehensive answer to this crucial question, an echocardiographic evaluation will be performed to assess the impact of CRT on LV remodelling in this patient population (secondary end point). Thus, a complete evaluation will be performed using clinical and remodelling assessments.

Finally, the use of a LV quadripolar lead in primary intention has been decided since it is currently used in French centres, thus enabling to achieve a crucial homogeneity of our population at baseline.

\section{CONCLUSIONS}

The NICD-CRT trial will add substantially to the modest amount of existing data on CRT in patients with NICD and should reduce uncertainty for guidelines and clinical practice when added to the pool of current information. 


\section{Author affiliations}

${ }^{1}$ Cardiology Department, Clermont Université, Université d'Auvergne, Cardio Vascular Interventional Therapy and Imaging (CaVITI), Image Science for Interventional Techniques (ISIT), UMR6284, and CHU Clermont-Ferrand, Clermont-Ferrand, France

${ }^{2}$ Hôpital Cardiologique du Haut-Lévêque, CHU Bordeaux, Université Bordeaux, IHU LIRYC, Bordeaux, France

${ }^{3} \mathrm{CHU}$ Clermont-Ferrand, Biostatistics unit (Clinical Research and Innovation Direction), Clermont-Ferrand, France

${ }^{4}$ Cardiology Department, Trousseau Hospital, François-Rabelais University, Tours, France

${ }^{5}$ North Hospital, Saint-Etienne, France

${ }^{6}$ Arrhythmia Unit, Cardiology Department, University Hospital, Grenoble, France

${ }^{7}$ Cardiology Department, Saint-Augustin Clinic, Bordeaux, France

${ }^{8}$ Cardiology Department, PHU2, Nantes University Hospital, Saint Herblain, France

${ }^{9}$ Nouvelles Cliniques Nantaises, Nantes, France

${ }^{10}$ Cardiology Department, Limoges University Hospital, Limoges, France

${ }^{11}$ Department of Cardiology and Vascular Disease, Pontchaillou Hospital, Rennes, France

${ }^{12}$ Federation of Cardiology, University Hospital Rangueil, Toulouse cedex 09, France

Acknowledgements The authors thank Mr Pierre Pothier for the editing of this manuscript and Miss Elodie Chazot for coordinating daily the NICD-CRT study.

Contributors RE, SP, BP, NC, ADC, PD, SG, J-BG, DG, BG-M, CL, PM and PB made substantial contributions to the conception, design of the work, acquisition, analysis or interpretation of data. RE, SP, BP, NC, ADC, PD, SG, J-BG, DG, BG-M, CL, PM and PB drafted the work and revised it critically for important intellectual content. RE, SP, BP, NC, ADC, PD, SG, J-BG, DG, $B G-M, C L, P M$ and $P B$ approved the final version of the manuscript to be published. RE, SP, BP, NC, ADC, PD, SG, J-BG, DG, BG-M, CL, PM and PB agreed to be accountable for all aspects of the work in ensuring that questions related to the accuracy or integrity of any part of the work are appropriately investigated and resolved.

Funding This clinical trial is funded by Boston Scientific (Cardiac Rhythm Management; 4100 Hamline Avenue North St. Paul, MN 55112; Kenneth Stein, MD, FACC, FHRS, Senior Vice President, Chief Medical Officer, CRM, kenneth.stein@bsci.com). However, the authors (under responsibility of the principal investigator of the NICD-CRT study: Romain Eschalier) are solely responsible for the design and conduct of this study as well as all study analyses and the drafting and editing of the article.

Competing interests None declared.

Patient consent Obtained.

Ethics approval Local ethics committee (CPP Sud Est VI, AU 1164) and ANSM (Agence Nationale de sécurité du medicament et des produits de santé, 2014-A01848-39) authorisations were obtained in March and May 2015 , respectively.

Provenance and peer review Not commissioned; externally peer reviewed.

Open Access This is an Open Access article distributed in accordance with the Creative Commons Attribution Non Commercial (CC BY-NC 4.0) license, which permits others to distribute, remix, adapt, build upon this work noncommercially, and license their derivative works on different terms, provided the original work is properly cited and the use is non-commercial. See: http:// creativecommons.org/licenses/by-nc/4.0/

\section{REFERENCES}

1. Cazeau S, Leclercq C, Lavergne T, et al. Effects of multisite biventricular pacing in patients with heart failure and intraventricular conduction delay. N Engl J Med 2001;344:873-80.

2. Abraham WT, Fisher WG, Smith $\mathrm{AL}$, et al. Cardiac resynchronization in chronic heart failure. N Engl J Med 2002;346:1845-53.

3. Moss AJ, Hall WJ, Cannom DS, et al. Cardiac-resynchronization therapy for the prevention of heart-failure events. $N$ Engl J Med 2009;361:1329-38.
4. Cleland JGF, Daubert JC, Erdmann E, et al. The effect of cardiac resynchronization on morbidity and mortality in heart failure. $N$ Engl J Med 2005;352:1539-49.

5. Sipahi I, Chou JC, Hyden M, et al. Effect of QRS morphology on clinical event reduction with cardiac resynchronization therapy: meta-analysis of randomized controlled trials. Am Heart $J$ 2012;163:260-7.e3.

6. Zareba W, Klein H, Cygankiewicz I, et al. Effectiveness of cardiac resynchronization therapy by QRS Morphology in the Multicenter Automatic Defibrillator Implantation Trial-Cardiac Resynchronization Therapy (MADIT-CRT). Circulation 2011;123:1061-72.

7. Baldasseroni S, Gentile A, Gorini M, et al. Intraventricular conduction defects in patients with congestive heart failure: left but not right bundle branch block is an independent predictor of prognosis. $A$ report from the Italian Network on Congestive Heart Failure (IN-CHF database). Ital Heart J 2003;4:607-13.

8. Baldasseroni S, Opasich C, Gorini M, et al. Left bundle-branch block is associated with increased 1-year sudden and total mortality rate in 5517 outpatients with congestive heart failure: a report from the Italian network on congestive heart failure. Am Heart $J$ 2002;143:398-405.

9. Wilensky RL, Yudelman P, Cohen Al, et al. Serial electrocardiographic changes in idiopathic dilated cardiomyopathy confirmed at necropsy. Am J Cardiol 1988;62:276-83.

10. Surawicz B, Childers R, Deal BJ, et al. AHA/ACCF/HRS recommendations for the standardization and interpretation of the electrocardiogram: part III: intraventricular conduction disturbances: a scientific statement from the American Heart Association Electrocardiography and Arrhythmias Committee, Council on Clinical Cardiology; the American College of Cardiology Foundation; and the Heart Rhythm Society. Endorsed by the International Society for Computerized Electrocardiology. J Am Coll Cardiol 2009;53:976-81.

11. Kashani A, Barold SS. Significance of QRS complex duration in patients with heart failure. J Am Coll Cardiol 2005;46:2183-92.

12. Wang NC, Maggioni AP, Konstam MA, et al. Clinical implications of QRS duration in patients hospitalized with worsening heart failure and reduced left ventricular ejection fraction. JAMA 2008;299:2656-66.

13. Zimetbaum PJ, Buxton AE, Batsford W, et al. Electrocardiographic predictors of arrhythmic death and total mortality in the multicenter unsustained tachycardia trial. Circulation 2004;110:766-9.

14. Bongioanni S, Bianchi F, Migliardi A, et al. Relation of QRS duration to mortality in a community-based cohort with hypertrophic cardiomyopathy. Am J Cardiol 2007;100:503-6.

15. Aro AL, Anttonen O, Tikkanen JT, et al. Intraventricular conduction delay in a standard 12-lead electrocardiogram as a predictor of mortality in the general population. Circ Arrhythm Electrophysiol 2011;4:704-10.

16. Eschalier R, Ploux S, Ritter P, et al. Nonspecific intraventricular conduction delay: definitions, prognosis, and implications for cardiac resynchronization therapy. Heart Rhythm 2015;12:1071-9.

17. Birnie $\mathrm{DH}, \mathrm{Ha}$ A, Higginson L, et al. Impact of QRS morphology and duration on outcomes after cardiac resynchronization therapy: results from the Resynchronization-Defibrillation for Ambulatory Heart Failure Trial (RAFT). Circ Heart Fail 2013;6:1190-8.

18. Gold MR, Thébault C, Linde C, et al. Effect of QRS duration and morphology on cardiac resynchronization therapy outcomes in mild heart failure: results from the Resynchronization Reverses Remodeling in Systolic Left Ventricular Dysfunction (REVERSE) study. Circulation 2012;126:822-9.

19. Cleland JGF, Mareev Y, Linde C. Reflections on EchoCRT: sound guidance on QRS duration and morphology for CRT? Eur Heart J 2015;36:1948-51.

20. Young JB, Abraham WT, Smith AL, et al. Combined cardiac resynchronization and implantable cardioversion defibrillation in advanced chronic heart failure: the MIRACLE ICD Trial. JAMA 2003;289:2685-94.

21. Beshai JF, Grimm RA, Nagueh SF, et al. Cardiac-resynchronization therapy in heart failure with narrow QRS complexes. N Engl J Med 2007;357:2461-71.

22. Goldenberg I, Moss AJ, Hall WJ, et al. Predictors of response to cardiac resynchronization therapy in the Multicenter Automatic Defibrillator Implantation Trial with Cardiac Resynchronization Therapy (MADIT-CRT). Circulation 2011;124:1527-36.

23. Khan FZ, Virdee MS, Palmer CR, et al. Targeted left ventricular lead placement to guide cardiac resynchronization therapy: the TARGET study: a randomized, controlled trial. J Am Coll Cardiol 2012;59:1509-18.

24. Signorini DF, Leung O, Simes RJ, et al. Dynamic balanced randomization for clinical trials. Stat Med 1993;12:2343-50. 
25. Brignole M, Auricchio A, Baron-Esquivias G, et al. 2013 ESC Guidelines on cardiac pacing and cardiac resynchronization therapy: the Task Force on cardiac pacing and resynchronization therapy of the European Society of Cardiology (ESC). Developed in collaboration with the European Heart Rhythm Association (EHRA). Eur Heart J 2013;34:2281-329.

26. Fuller BM, Ferguson I, Mohr NM, et al. Lung-protective ventilation initiated in the emergency department (LOV-ED): a study protocol for a quasi-experimental, before-after trial aimed at reducing pulmonary complications. BMJ Open 2016;6:e010991.

27. Hashmi F, Torgerson D, Fairhurst C, et al. EVerT2-needling versus non-surgical debridement for the treatment of verrucae: study protocol for a single-centre randomised controlled trial. BMJ Open 2015;5:e009406.

28. Aranda JM, Conti JB, Johnson JW, et al Cardiac resynchronization therapy in patients with heart failure and conduction abnormalities other than left bundle-branch block: analysis of the Multicenter InSync Randomized Clinical Evaluation (MIRACLE). Clin Cardiol 2004;27:678-82.

29. Goldenberg I, Kutyifa V, Klein HU, et al. Survival with cardiac-resynchronization therapy in mild heart failure. N Engl J Med 2014;370:1694-701.

30. Rickard J, Kumbhani DJ, Gorodeski EZ, et al. Cardiac resynchronization therapy in non-left bundle branch block morphologies. Pacing Clin Electrophysiol 2010;33:590-5.
31. Ploux S, Lumens J, Whinnett Z, et al. Noninvasive electrocardiographic mapping to improve patient selection for cardiac resynchronization therapy: beyond QRS duration and left bundle branch block morphology. J Am Coll Cardiol 2013;61:2435-43.

32. Ploux S, Eschalier R, Whinnett ZI, et al. Electrical dyssynchrony induced by biventricular pacing: implications for patient selection and therapy improvement. Heart Rhythm 2015;12:782-91.

33. Cleland JG, Abraham WT, Linde C, et al. An individual patient meta-analysis of five randomized trials assessing the effects of cardiac resynchronization therapy on morbidity and mortality in patients with symptomatic heart failure. Eur Heart $J$ 2013;34:3547-56

34. Epstein AE, DiMarco JP, Ellenbogen KA, et al. 2012 ACCF/AHA HRS Focused Update Incorporated Into the ACCF/AHA/HRS 2008 Guidelines for Device-Based Therapy of Cardiac Rhythm Abnormalities A Report of the American College of Cardiology Foundation/American Heart Association Task Force on Practice Guidelines and the Heart Rhythm Society. Circulation 2013;127: e283-352.

35. Sun H, Davison BA, Cotter G, et al. Evaluating treatment efficacy by multiple end points in phase II acute heart failure clinical trials: analysing data using a global method. Circ Heart Fail 2012;5:742-9. 